\title{
Effect of Quality Improvement Approaches on Prevention of Mother to Child Transmission Services Coverage in Nairobi County
}

\author{
Duece N. Malava *, Peterson Muriithi, Chrisostim Barasa \\ School of Public Health, College of Health Sciences, University of Nairobi, Nairobi, Kenya \\ *Corresponding author: Duece N. Malava; dueceochieng@gmail.com
}

Received 19 April 2021;

Accepted 14 May 2021;

Published 22 May 2021

\begin{abstract}
Background: Improving the quality of healthcare is a growing international concern as it ensures that the healthcare system functions efficiently. Quality improvement in the HIV field focuses on achieving essential health outcomes, including patient retention, increasing viral load suppression, and improving overall health outcomes of people living with HIV. Quality Improvement (QI) approaches form part of the global strategies recommended by WHO to improve prevention of mother to child transmission (PMTCT) coverage and to achieve virtual elimination of mother to child transmission (MTCT). Nairobi County is implementing QI approaches using the Kenya Quality Model for Health (KQMH) framework. The implementation of QI began in January 2016, and since then, the facilities have formed work improvement teams (WITs) that work to improve the quality in the facilities. Study objective: This study sought to evaluate the effect of quality improvement approaches on PMTCT coverage in Nairobi County. Methodology: This quasi-experimental study applied a mixed-method research methodology. Purposive sampling determined the four study facilities. The participants were women attending the PMTCT clinic at the selected facilities, identified through a purposive sampling process. DHIS reports and facility registers provided the necessary quantitative data, while Focus Group Discussions (FGDs) and Key informant interviews (KIIs) provided qualitative data. Quantitative data showing service delivery uptake over time provided a trend on the performance of the key indicators under study. This data analysed using an interrupted time series approach as well as by using descriptive statistics showed trends over time. Qualitative data assessed clients' experiences while accessing PMTCT services, providing key insights from the clients' perspective. The researcher used content analysis to analyse this data. $\underline{\text { Results: }}$ The study found out that quality improvement is an integral part of PMTCT service delivery. Through the FGDs, the PMTCT services provided generally satisfy the clients' needs, and that the clients have a significant role in quality improvement. The HCWs can implement QI approaches as long as they have the facility management's support and leadership. Conclusion: The study found out that quality improvement in PMTCT relies heavily on the systems being in place and on teamwork between the HCWs and clients as they access treatment services at the health facilities.
\end{abstract}

Keywords: Quality Improvement (QI), PMTCT, Patient Satisfaction, Continuous Quality Improvement (CQI), Kenya.

\section{Introduction}

Globally, mother to child transmission (MTCT) is the primary mode of HIV acquisition in children. The MTCT can occur during pregnancy, childbirth, or breastfeeding period ${ }^{[29]}$. Before the development of effective interventions to reduce MTCT of HIV, the estimated transmission rates were $25 \%-40 \%$ among women who are breastfeeding in resource-limited countries and 15\%-25\% among women who are not breastfeeding in North America and Europe $^{[4]}$.

In 2017, 1.5 million people were living with HIV in Kenya. The percentage of women living with HIV was higher than that of men at $5.2 \%$ and $4.5 \%$ respectively. The rate of new infections among people aged 15-49 has reduced from $0.35 \%$ in 2010 to $0.19 \%$ in 2017 with a prevalence rate of $4.85 \%{ }^{[22]}$. This group represents the reproductive age group in which PMTCT would be most critical. The coverage for PMTCT services has increased from $23 \%$ in 2005 to $76 \%$ in 2017 while the MTCT rate reduced over the same period from $29.7 \%$ in 2005 to $11.7 \%$ in $2017^{[22]}$.

A four-prong strategy is required to make PMTCT more effective. The first prong focuses on preventing HIV infection among potential parents by ensuring HIV testing and other prevention interventions are available to them. Ensuring that HIV positive women avoid unwanted pregnancies by providing appropriate counselling on contraception forms the second prong. The third prong advocates for the use of antiretroviral therapy for all HIV positive pregnant and breastfeeding women to prevent HIV transmission. The fourth prong focuses on providing appropriate care, support, and treatment to mothers living with HIV and their children and families ${ }^{[20]}$. 
Nairobi County has been implementing prevention of mother to child transmission of HIV (PMTCT) since 2000, with a scale-up of services occurring over the years. As part of the WHO PMTCT strategic vision 2010 - 2015, improving the quality and delivery of PMTCT services is a crucial step in addressing the gaps identified in PMTCT ${ }^{[29]}$. Through the support of the National AIDS and STI Control Program (NASCOP) and various implementing partners, health care facilities began implementation of quality improvement approaches. Within the health facilities, implementation of QI occurs across the different departments, including PMTCT, to improve the quality and delivery of services.

Quality has been defined as "the totality of features and characteristics of a product or service that bears its ability to satisfy stated or implied needs" ISO 8402-1986. The American Society of quality defines it as "The characteristics of a product or service that bear on its ability to satisfy stated or implied need". Quality can also be a product or service free of deficiencies". According to the Classical theory in quality management and improvement, the key principles of quality are customer focus, continuous improvement, process orientation, teamwork, and decisions based on facts ${ }^{[25]}$.

Generally, the act of "doing better" is what is considered as an improvement ${ }^{[2]}$. Quality Improvement (QI) involves doing systematic and continuous actions that lead to improved health care services and the health status of targeted patient groups ${ }^{[9]}$. Quality improvement (QI) in healthcare consists of varied models and methods, which aim to improve healthcare, to make healthcare more effective and efficient and to increase safety index for the patients ${ }^{[6]}$. QI seeks to improve health system performance.

The Institute of Medicine defines quality in healthcare as the "degree to which healthcare services offered to the population or individuals increase the likelihood of the desired health outcomes and are consistent with current professional knowledge" ${ }^{[10]}$. It further says that six domains provide the criteria by which to judge healthcare quality. These domains are effective, safe, equitable, efficient, timely, and patient-centered. According to Gupta and Rokade ${ }^{[7]}$, patient satisfaction is a critical feature of quality healthcare. Healthcare quality is essential because it is indicative of the functioning of the health system.

A growing international concern of the health sector is on ensuring that there is an improvement in the efficiency in service delivery. According to the World Health Organization (WHO), inefficiency accounts for wastage of $20 \%-40 \%$ of resources spent on health ${ }^{[30]}$. While scaling up of essential health coverage at a global scale requires more resources, improvements in health could be made with available resources if countries worked to improve their healthcare systems efficiency ${ }^{[30]}$.

Improving the healthcare system is crucial because it increases the chances of patients achieving the expected outcomes and benefits of care. Quality Improvement use in other service delivery areas of healthcare show successful implementation. Studies show how QI was used to improve child health services ${ }^{[3]}$, maternity services ${ }^{[11,19]}$ as well as in improving the cost of healthcare service delivery ${ }^{[31]}$. Quality Improvement is also important in combating the HIV/AIDS pandemic, as well as in meeting the goals of the National HIV/AIDS Strategy. With the implementation of QI approaches, indicators like HIV care, linkage, and retention can be improved ${ }^{[14]}$. Quality Improvement within the HIV field focuses on achieving crucial health outcomes that include overall retention in care, increasing viral load suppression, and improving the general health outlook of HIV positive individuals ${ }^{[14]}$.
The objective of QI in PMTCT is to improve the services delivered to PMTCT clients, which would ideally lead to efficient services, customer satisfaction, and improved outcomes. Achieving virtual elimination of transmission of HIV from mother to child is the goal of PMTCT services in Kenya. Quality improvement is required in all the four prongs of PMTCT and needs dedicated teams to implement the approaches. Implementation of QI in PMTCT has shown success in improving PMTCT in several areas of implementation ${ }^{[1,15,18,32]}$.

Kenya launched the Kenya Quality Model for Health (KQMH) in 2012. The Kenya Quality Model for Health (KQMH) is a conceptual framework intended to provide an integrated approach to improve the quality of healthcare. KQMH is the revised version of the Kenya Quality Model (KQM), introduced in 2001 and piloted in 2003. The need to align the model to the newly introduced Kenya Essential Package of Care (KEPH) levels of care led to the revision of KQM. KQMH incorporates evidence-based medicine with total quality management (TQM) and patient partnerships ${ }^{[27]}$. The main aim of KQMH is to improve adherence to standards and guidelines that are evidence-based, as well as apply quality principles and tools to improve structure, process, and outcomes. It also aims to ensure client satisfaction in a culturally acceptable way. Implementation of KQMH is the first step towards ISO certification. The first step in the implementation of KQMH begins with involving top leadership to get buy-in for the process. Involving senior management is crucial, as their leadership is required to implement quality improvement approaches.

Developed in 2014 and adopted from KQMH, the Kenya HIV Quality Improvement Framework (KHQIF) is a framework meant to guide the implementation of HIV quality improvement ${ }^{[21]}$. The main goal of KHQIF is to ensure that there is the provision of quality HIV services, to improve patient and HIV program outcomes, to improve the standard of living among people living with HIV, to prevent new HIV infections, and to reduce MTCT in Kenya. Implementation of KHQIF is through the Plan-Do-StudyAct (PDSA) and 5S (sort, set, shine, standardize, and sustain) models, which form the backbone for the Total Quality Management (TQM). The TQM model has proven to be ideal for continuous improvement activities for services that involve patients with chronic conditions ${ }^{[14]}$.

The success of KHQIF implementation relies on measuring performance at every level. Performance measurement involves the development of set indicators, collecting data on these indicators using appropriate tools, and analysing this information to determine the performance ${ }^{[14]}$. In PMTCT, these indicators focus on retention of mother-baby pairs in care, uptake of essential PMTCT services like HIV testing and ART initiation as well as viral suppression.

Nairobi County embarked on improving the quality of PMTCT services using the KQHIF model since January 2016. The process began with buy-in from the County and Sub County teams before cascading down to the health facilities. In the beginning, the County teams, Sub-county teams, as well as facility-level teams received sensitization on KQHIF, which paved way for the identification of QI champions at each level. The facilities then set up Quality Improvement teams (QITs) and Work Improvement Teams (WITs) whose mandate was to identify areas of improvement within the different service delivery areas.

The QI strategy used involved forming quality improvement teams (QITs) and Work improvement teams (WITs) within the facilities. QITs are responsible for the overall quality of the facilities and work to ensure there is coordination and 
implementation of QI activities within the facilities. They are composed of the Health Facility Management Team and middle management, as well as heads of departments. WITs are specific to departments in which the staff work, and its members are drawn from the service areas and support departments. Each team is composed of between 3 to 15 members of staff. The teams meet once or twice a month and work to identify gaps in service delivery. Upon identification, the teams conduct a root cause analysis to come up with change ideas to address those gaps. The WITs use the Plan-Do-Study-Act (PDSA) cycle and 5S (sort, set, shine, standardize, and sustain) to implement quality improvement approaches in their service delivery areas.

The conceptual framework for the study was derived from the Total Quality Management (TQM) as enshrined in the KHQIF. TQM is a quality improvement approach that focuses on providing customer satisfaction ${ }^{[8]}$. The critical aspect of quality healthcare is customer care $^{[7]}$. In TQM, maintenance of customer satisfaction is

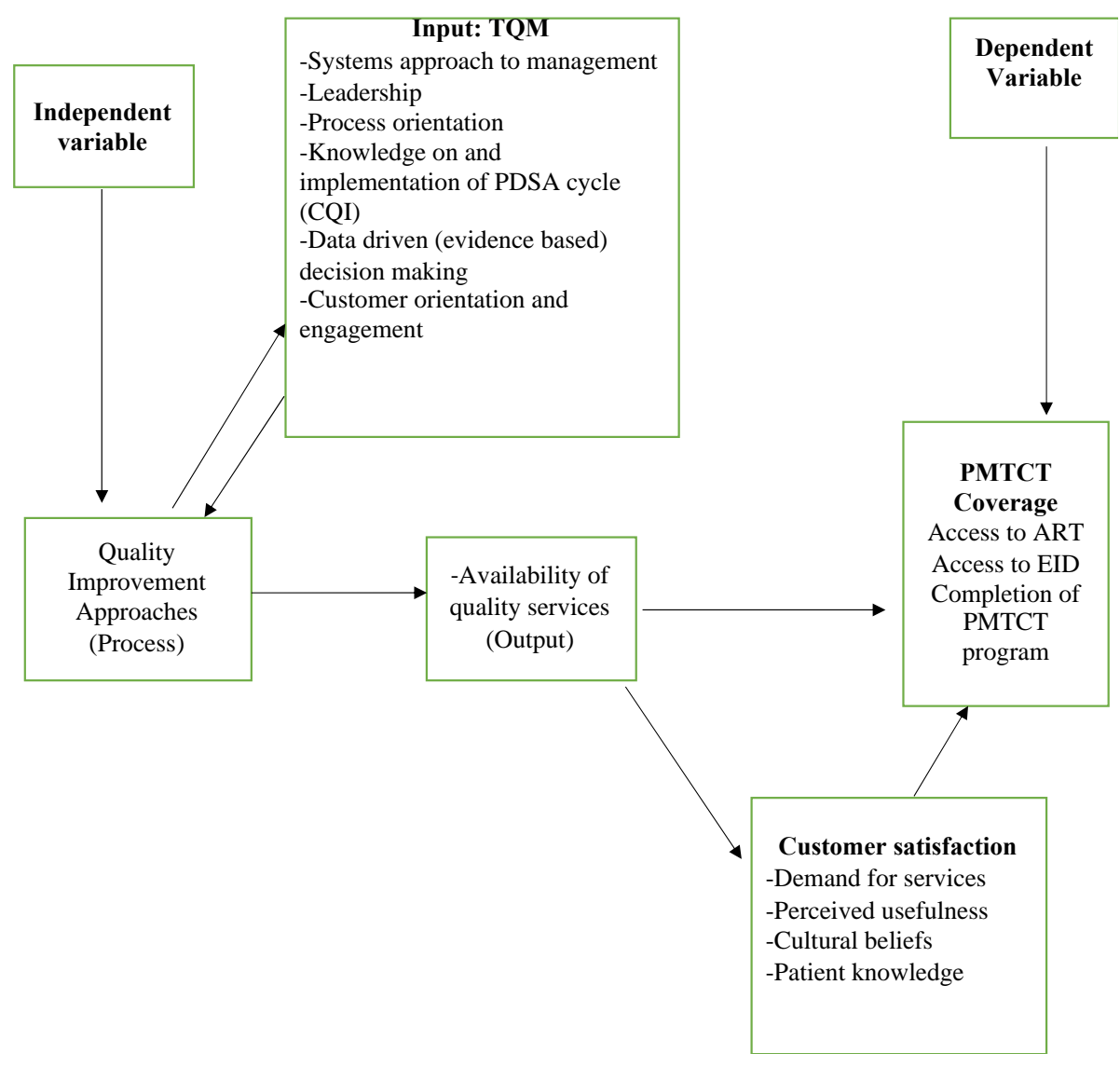

Figure 1: Conceptual framework of study (Adapted from TQM based on the KHQIF model)

\section{Materials and Methods}

\section{Study Design}

The study was a quasi-experimental study design. The researcher chose this design because the study sought to describe the existence of a causal relationship between the research variables rather than confine it to data collection only. It sought to get a view of the situation before the initiation of PMTCT QI approaches in the selected facilities by comparing the performance of PMTCT quality indicators for the period before implementation of QI approaches with the performance after QI implementation to determine effect.

A mixed-method research design ${ }^{[16]}$ determined the effect of QI on PMTCT coverage. The qualitative aspect of the design offered a chance to the consumers of the PMTCT services to provide their views. The clients' experiences complemented the by ensuring that the functions, culture, and services of an organization are all integrated ${ }^{[28]}$. Customer feedback and input provides a critical piece in ensuring that services meet their needs. An organization achieves TQM by continuously ensuring that all members actively participate in improving the processes in their areas of work. TQM focuses on continuous quality improvement, intending to improve results, and improving the capabilities that will produce better results in the future. Under KHQIF, TQM has six principles (inputs), which are a systems approach to management, leadership, process orientation, knowledge on and implementation of PDSA cycle (CQI), data-driven (evidencebased) decision making, and customer orientation and engagement. These inputs guide the QI approaches (process) to provide quality services (output). An organization can have all six principles in place without taking into consideration the QI process, and this would harm the provision of quality services. study results and served to strengthen the study's conclusion. Focus Group Discussions and Key Informant Interviews that allowed the study participants to share their experiences openly provided the qualitative data for the study. A review of the secondary data in the form of registers and facility reports from the selected facilities provided the quantitative data. This provided information on the status of services before and after the implementation of QI approaches in PMTCT service delivery and helped to cement the findings from the qualitative data. The data extracted from the registers and facility reports included data on PMTCT indicators namely HIV testing rates, ART uptake among pregnant and breastfeeding women, as well as the retention rates among the mother-baby pair. The extracted data helped in determining trends over time and provided clarity on the performance of the PMTCT indicators under study. 


\section{Study Area}

Nairobi County is the capital city of Kenya, covering an area of 696 square kilometres. Nairobi County lies at an altitude of 1,798 meters above sea level, and it borders Kiambu County to the North and West, Kajiado to the South, and Machakos County to the East. There are ten sub-counties in Nairobi County, which are Embakasi East, Embakasi West, Dagoretti, Kamukunji, Langatta, Kasarani, Ruaraka, Makadara, Starehe, and Westlands Sub-counties. According to the Kenya National Bureau of Statistics projections in 2019 , the population of Nairobi County is $4,397,073$, out of which $49 \%$ are males while $51 \%$ are females. Children make up $34 \%$ of the total population ${ }^{[12]}$.

Nairobi County has approximately 968 health facilities, out of which 139 are public health facilities, 108 are NonGovernmental Organizations owned facilities, 103 are faith-based, and 618 are privately owned health facilities ${ }^{[13]}$. These facilities receive pharmaceutical and non-pharmaceutical supplies from KEMSA. The facilities hold regular meetings within themselves and with the sub-county teams to determine progress and address any identified gaps.

With an HIV prevalence rate of $6.1 \%$, Nairobi County is contributing to $11.3 \%$ of the cumulative number of people living with HIV in Kenya. The prevalence of HIV in men is lower at $4.7 \%$ than that of women at $7.5 \%$. In 2018 , the county contributed to $4 \%$ of new infections in children and $7 \%$ of new infections in adults in Kenya. Nairobi County has had significant interventions to help reduce transmission of HIV from mother to child, including rolling out of Option B+, Beyond Zero, and Bring Back the Mothers Campaign. Through these interventions, coverage of PMTCT increased to $97 \%$, while MTCT rates stood at $3.7 \%$ in $2015^{[22]}$. The researcher chose Nairobi County for the study because the implementation of quality improvement approaches particularly in PMTCT services had not been evaluated before.

\section{Study Sites}

The sites targeted for the study were Mama Lucy Kibaki Hospital, Mbagathi Hospital, Westlands Health Centre, and Kangemi Health Centre. The researcher chose these facilities because they are high CCC patient volume sites in Nairobi County. High volume facilities represent those facilities that have more than 1000 clients enrolled in the $\mathrm{CCC}$, which in turn provided the capacity to handle PMTCT clients. Another selection criterion was that the facilities chosen provided integrated ANC and PMTCT services in the MCH clinic for eligible clients and were able to provide follow up until discharge from the PMTCT program. These MCH clinics receive more than $50 \mathrm{ANC}$ clients per day and have the capacity to handle PMTCT clients. To qualify for the study, PMTCT should have been operational for more than six months before the implementation of the QI strategy. As a policy, these facilities should have a functional QIT that oversees the implementation of QI projects in PMTCT. Thus, having a QIT/WIT that was actively undertaking QI projects in PMTCT qualified a site for the study.

These facilities have facility in-charges who provide overall facility management. Within each site, there are different departments, which include the maternal and child health $(\mathrm{MCH})$ clinics where PMTCT clients receive services. Departmental heads, who are mainly qualified nurses, lead the various departments in the health facilities while the QIT/WIT team leaders head the QIT/WITs in their facilities. The QIT/WIT leads have received training on QI implementation and provided overall leadership for QI implementation in their teams.

These facilities have qualified health care workers working in the $\mathrm{MCH}$ clinics who undergo regular refresher training and on the job training to improve their knowledge and capacity in the management of PMTCT clients. They produce monthly reports showing the performance of the different PMTCT indicators and use these reports to guide decision making during service delivery. Each site has a customer desk and a suggestion box to allow clients to provide feedback on the services received.

\section{Study Participants}

The members of the FGDs came from women seeking PMTCT services at the selected facilities, which included women making initial and follow-up ANC visits, as well as mother-infant pairs on follow-up. Recruitment of the FGD participants occurred as they came for their regular clinic appointments and depended on their willingness to participate. Departmental heads, as well as PMTCT WIT team leaders, formed the target group for the key informant interviews, as their insight was crucial to the study.

\section{a) Inclusion Criteria}

The facilities included in the study were high volume facilities with more than 1000 clients in the CCC. They also have integrated PMTCT in the MCH clinic that caters to 50 clients or more daily. They also had a functional WIT actively undertaking QI projects.

Those recruited to participate in the FGDs were clients seeking PMTCT services in the facilities at the time of the study. These clients had come to the facility more than once and were present when the QI interventions took place. To take part in the KIIs, one had to be a team leader of the PMTCT WITs or head of department in the $\mathrm{MCH}$ in each facility. They also needed to be conversant with the QI projects undertaken by their teams.

\section{b) Exclusion Criteria}

Lack of a functional QIT/WIT excluded a site from the study. The study also excluded privately managed facilities as well as those that have not integrated PMTCT services in the $\mathrm{MCH}$ clinic. Additionally, those facilities with a QIT/WIT but not having an active QI project at the time of study did not participate.

Clients who came to the facility to access other services, including the very sick clients, did not participate in the FGDs. The study excluded the clients on transit and those who had not been enrolled in the PMTCT clinics at the facilities. Other members of the PMTCT WIT or other departmental WIT leaders did not take part in the KIIs.

\section{Assumptions for Study Participants}

The study made several assumptions. The first assumption was that the population served in the selected facilities was homogenous. A Homogenous population refers to one, which has the same characteristics and corresponds in structure because of a common origin. The study also assumed that there was control over the moderating variables, which means there was no influence over the dependent and independent variables. The third assumption made was that there were no significant policy changes implemented during the period of interest that would have otherwise affected the outcomes of PMTCT.

\section{Sample Size}

The study selected four high volume health facilities in Nairobi County using the criteria mentioned above. Each facility had one working group team. In each facility, the study targeted departmental heads/facility head, QIT/WIT team leaders, and PMTCT clients. Clients accessing PMTCT services in each facility had one FGD. Key Informant Interviews targeted the QIT/WIT team leaders and $\mathrm{MCH}$ departmental heads. In total, the researcher conducted four FGD and four KIIs. Each facility has registers and 
records showing the performance of the key PMTCT indicators that were also reviewed as part of the study.

Table 1: Sample size distribution of study participants

\begin{tabular}{|l|l|}
\hline Target group & Sample size \\
\hline FGD teams (made up of 10 members) & 4 groups \\
\hline WIT team leaders or Departmental head & 4 \\
\hline
\end{tabular}

\section{Sampling Technique}

The four study facilities were purposely selected using the criteria mentioned above. Those not meeting the selection criteria were not part of the study. The participants who took part in the focus group discussion were also selected using the purposive sampling technique. The selection of participants, in this case, targeted those clients who were accessing PMTCT services at the time of study and were willing to take part in the FGD. To identify the specific clinic days with a high client load, the researcher used the appointment management system available in the chosen PMTCT clinics. Once identified, the researcher enrolled the participants to the FGD as the clients accessed services depending on their willingness to participate. The QIT/WIT team leaders or departmental heads $(\mathrm{MCH})$ took part in the KIIs because their insight was very crucial to the study.

\section{Other Study Procedures}

All the participants who took part in the study received detailed information about the research before providing informed consent for participation. During the consenting process, the researcher went through the consent form with the study participants, explaining crucial details in the consent form. Once finished, the researcher allowed the participants to seek clarification before proceeding to sign the consent forms. No participant took part in the study before signing the consent forms.

In order to conduct the review of facility reports and registers, the researcher provided detailed information to the facility management and the records department before seeking consent to retrieve the records and registers. The review period for this was from July 2014 to June 2019. No records were reviewed without prior consent from the facility management.

\section{Study Variables}

The following were the study variables investigated in the study under each objective, derived from the conceptual framework.

Table 2: Study Variables as derived from the conceptual framework

\begin{tabular}{|l|l|l|l|}
\hline Objective & Variable & Definition of variables & Data Source \\
\hline $\begin{array}{l}\text { Objective1: To identify the factors influencing the } \\
\text { implementation of quality improvement approaches }\end{array}$ & $\begin{array}{l}\text { PMTCT service delivery } \\
\text { factors }\end{array}$ & Intermediate variable & $\begin{array}{l}\text { FGDs from PMTCT clients } \\
\text { Interviewer guided KIIs }\end{array}$ \\
\hline $\begin{array}{l}\text { Objective 2: To determine the influence of quality } \\
\text { improvement on the uptake of PMTCT services }\end{array}$ & $\begin{array}{l}\text { Monitoring and evaluation } \\
\text { of PMTCT indicators }\end{array}$ & Intermediate variable & $\begin{array}{l}\text { Facility PMTCT registers } \\
\text { Facility records }\end{array}$ \\
\hline $\begin{array}{l}\text { Objective 3: To determine the influence of quality } \\
\text { improvement approaches on retention to care }\end{array}$ & $\begin{array}{l}\text { Client retention in PMTCT } \\
\text { program }\end{array}$ & Intermediate variable & $\begin{array}{l}\text { Facility PMTCT registers } \\
\text { Facility records } \\
\text { Client feedback (FGDs) }\end{array}$ \\
\hline
\end{tabular}

\section{Data Collection}

The study collected both primary and secondary data reported from the facility. FGDs and Key Informants Interviews provided the primary data. The focused group discussions carried out in all the four facilities targeted eight to ten participants. The FGDs sought to understand the clients' perspective when it came to quality and helped determine the level of satisfaction in the services provided. Key informant interviews were done with QIT/WIT team leaders or MCH departmental heads, allowing them to get their views on the QI process. The researcher developed an Interview Guide for the Key Informant Interviews and a Focused Group Discussion guide for the FGDs to collect data. A digital voice recorder recorded the discussions during FGDs to supplement the notes recorded by the moderator during the group discussions. All the participants consented to the use of a digital voice recorder to record the sessions.

A review of facility registers and DHIS reports provided the needed secondary data. The data collected from the facility registers and DHIS included data on HIV testing rates, ART uptake among pregnant and breastfeeding women, as well as the retention rates among the mother-baby pair. The data collection focused on the period before implementation of quality improvement (October 2014 to September 2015) and the period after quality improvement (October 2018 to July 2019). The collected data helped in determining trends over time, which was crucial to make inferences in the study.

\section{Data Management and Analysis}

The data sets collected from the register reviews and DHIS reporting were edited, cleaned, and tabulated, with data entered into statistical software, namely Statistical Package for Social Sciences Program (SPSS) for analysis. Data cleaning eliminated any errors within the data sets and allowed the researcher to make conclusions from the data. Transcription of the data collected by the voice recorder used during the FGDs produced a written text. The researcher analysed this text alongside field notes documented by the moderator of the discussion. The information collected using the KIIs underwent cleaning and coding to draw conclusions from it. All the data collected were stored in well-labelled and dated files with separate folders for each data set for easy access. Electronic data storage in computers secured by passwords avoided unauthorized access to the data sets. Data backup occurred electronically.

\section{a) Quantitative Data Analysis}

An interrupted time-series design that compared performance before and after QI approaches provided the approach to data analysis. The researcher used descriptive statistics which included frequencies and percentages to analyse quantitative data and presented this in the form of line and bar graphs. Graphical presentation of data showed the trends in the PMTCT cascade, mainly looking at HIV testing trends, ART initiation among identified positives, early infant diagnosis, and retention rates among the mother-baby pair. Chi-square $\left(\chi^{2}\right)$ inferential statistical technique further analysed this data to determine any association between the independent and dependent variables. The independent variable defined the use of quality improvement approaches, while the dependent variable defined coverage and utilization of PMTCT services.

\section{b) Qualitative Data Analysis}

The researcher used content analysis to analyse qualitative data. Analysis of FGD data involved going through both the field notes and recorded sessions to get transcribed text. The researcher went 
through this text to identify key categories of words or phrases that address the areas of interest. This allowed the researcher to code the information along with the recurring themes on the clients' feedback. The researcher then transcribed the data collected by the KII guides and reviewed this text to pick out any recurring themes, which underwent coding. KIIs provided the healthcare workers' perspective on quality improvement.

\section{Results and Discussion}

This chapter describes the study findings as obtained from the study participants and review of secondary data. Data collection occurred in the period between June and July 2019. The presentation of the results is in line with the study objectives.

\section{Study Flow}

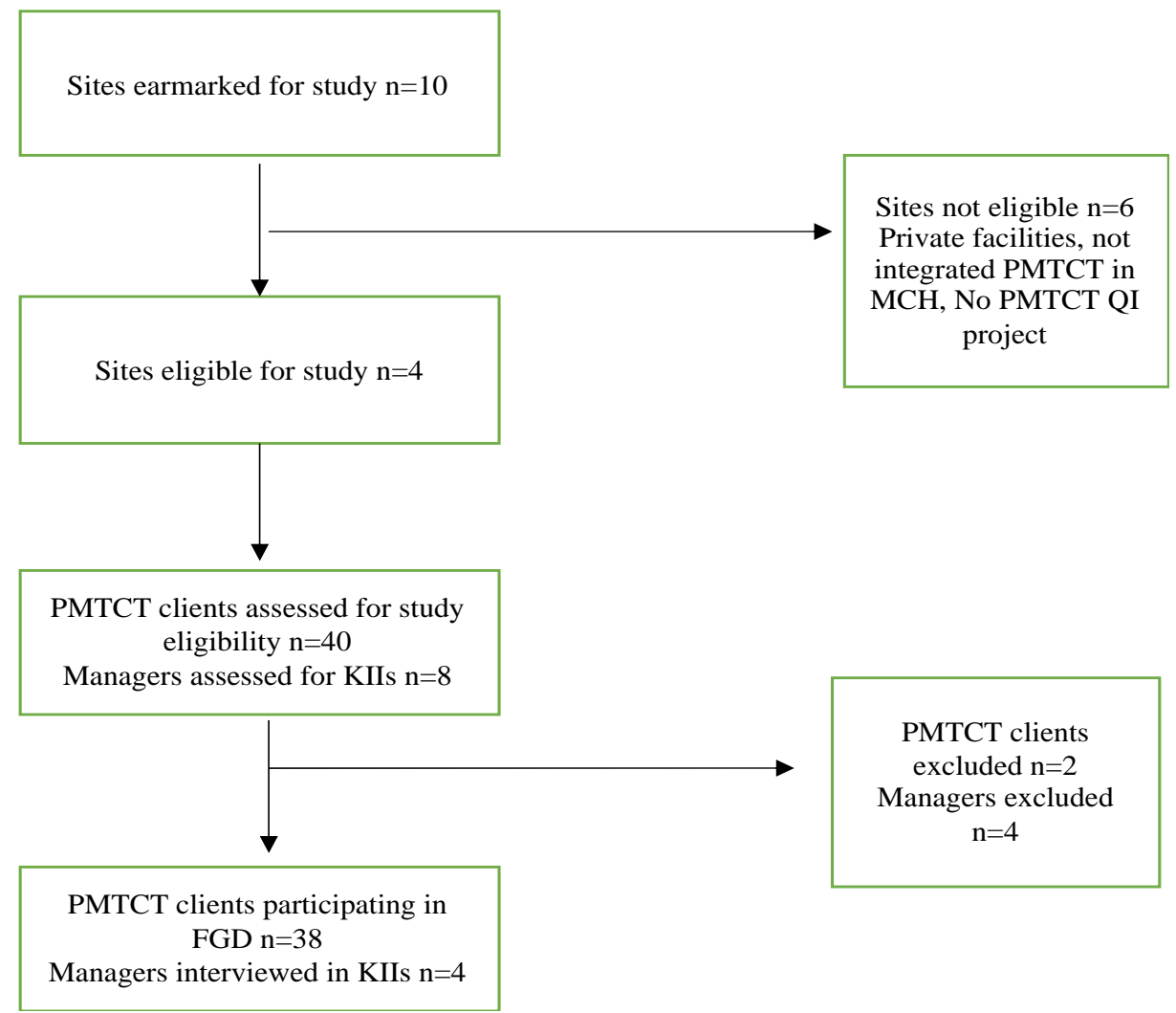

Figure 2: Study Flow

\section{Socio-Demographic Data of Study Participants}

\section{a) Focus Group Discussion Study Participants}

The table below shows the age brackets of the study participants of the FGDs. The results show that majority of the study participants
(71\%) were in the age bracket 25-49 years, while the rest (29\%) were in the 18-24 age bracket. These results show that the clients seeking PMTCT services fall within the reproductive age group in which PMTCT would be most critical.

Table 3: Sociodemographic data of FGD study participants

\begin{tabular}{|l|l|l|l|}
\hline Health Facility & Total FGD participants & $\mathbf{1 8 - 2 4}$ years & 25-49 years \\
\hline Mama Lucy & 10 & 3 & 7 \\
\hline Mbagathi & 8 & 0 & 8 \\
\hline Kangemi & 10 & 6 & 4 \\
\hline Westlands & 10 & 2 & 8 \\
\hline & $\mathbf{3 8}$ & $\mathbf{1 1}(\mathbf{2 9 \%})$ & $\mathbf{2 7}(\mathbf{7 1 \%})$ \\
\hline
\end{tabular}

\section{b) Key Informants Interview Study Participants}

This information comprises the cadres of staff, work experience, qualification, and level of involvement in work improvement teams. As shown from the table, the least work experience by the staff members was below two years, with the highest being five years. The highest qualification was a Master's degree level while the lowest was a diploma level.
In one of the study facilities, where the in-charge had a Masters level, other cadres of staff had more involvement in implementing Quality Improvement approaches mainly because the in-charge was involved actively in other duties, thereby shifting this task to other members of staff.

Table 4: Sociodemographic data of KII study participants

\begin{tabular}{|l|l|l|l|}
\hline Position & No of years worked at the facility & Qualifications & Level of involvement in the work improvement team \\
\hline PMTCT clinician & $3-5$ & Diploma & High \\
\hline MCH In charge & $3-5$ & Masters & Moderate \\
\hline PMTCT nurse & Below 2 years & Diploma & High \\
\hline PMTCT clinician & Below 2 years & Diploma & High \\
\hline
\end{tabular}




\section{Factors Influencing the Implementation of Quality}

\section{Improvement approaches}

The key highlights on the views of the facility managers on quality improvement came about during the KIIs. All the facilities under study had both Quality Improvement Teams (QITs) to undertake overall quality improvement for the facilities and Work Improvement Teams (WITs) in the PMTCT clinic to cater to quality improvement. The standard package of care offered in the facilities included ART, prophylaxis for the infant, partner and family testing services, as well as adherence counselling. Other services included STI screening and management, post-abortion care, focused antenatal care, treatment monitoring, nutritional counselling, as well as referrals for additional support as needed. This is in line with the four-pronged approach to PMTCT as recommended by WHO.

Several factors influenced the implementation of quality improvement interventions. These included the identified gaps in service delivery, staff shortage, and heavy workload, which limits the ability to conduct meetings, lack of co-operation between the teams (staff and clients) affecting teamwork and client involvement in QI. Other factors influencing QI were the presence of support from facility management and the availability of data to identify performance gaps as well as monitor performance against targets. The KIIs identified several challenges affecting quality PMTCT service provision including lack of space to offer privacy, lack of adequate supplies including ART, poverty among the clients, stigma from the clients related to HIV services, violence among partners affecting PMTCT uptake and lack of knowledge among clients affecting health seeking behaviours.

The responses from the KIIs showed that all the study facilities use standard tools to measure quality improvement. These tools are HIV-exposed infant cards, viral load tracking register, data abstraction tools and minutes taken during the work improvement meetings. Other tools used to measure QI are run charts, PDSA graphs, client exit forms, and data review minutes. All these tools are important in determining the progress of quality improvement interventions.

The study respondents felt that management plays a critical role in ensuring the implementation of quality improvement. This includes providing support supervision, ensuring adequate supplies, and commodities for service provision, ensuring that staff are capacity built and receive regular updates in their service areas, providing good governance as well as continuous monitoring and reviewing QI projects. Additionally, the management should ensure that all facility staff are involved in quality improvement and should strive to support the facility teams in QI implementation. This addresses the role of leadership in quality improvement.

According to the KII respondents, PMTCT clients also have a role in quality improvement by honouring clinical appointments, as well as adhering to their treatment and other instructions. Additionally, the PMTCT clients have a role in providing feedback to facility staff on the care they have received as well as being cooperative with their service providers. The clients get to learn about this during health talks given to them as they wait to receive their services.

Based on their experiences with the health facilities, the clients had very practical recommendations on how to improve the services offered at the PMTCT clinics. The recommendations point to the interventions that need to be in place for the consumer to feel satisfied with the services received. The participants at Mama Lucy Kibaki Hospital recommended that the facility introduce a heater at the triage section, which is very cold risking the health of the children. They also recommended the expansion of maternity services due to the congestion experienced. This congestion forces mothers to share beds and in some cases because of this, some do not get a chance to sleep as seen in the quote below:

"Yes, and some patients share beds such that those who are operated are the only ones allowed to sleep but the rest you have to sit even after delivering." Source: Mama Lucy Kibaki Hospital FGD participant.

At Westlands, to minimize delays over breaks the participants recommended the introduction of shifts for the staff. The participants also noted that there is need to hire additional staff, as the current ones are overwhelmed causing long delays. Similarly, most participants recommended an increase in the supply of drugs across all the four facilities. Patient discrimination also needs addressing. A participant from Kangemi FGD had this to say about patient discrimination:

"They should not discriminate people such that if you came at whatever time, you should follow the line as other people also want to be attended to. Although the old ones and emergencies can be attended to first, but the rest should just follow the line." Source: Kangemi Health Centre FGD participant.

The participants also felt that their input in quality improvement meetings was important as it provides an avenue to give their opinions with regards to quality. At Mama Lucy Kibaki Hospital, they thought it is good to have a patient representative in such meetings to give feedback particularly in mistreatment cases. At Westlands, the participants opined that such meetings would provide opportunities to address and solve conflicts, give suggestions, and build a team spirit. In the quote below, a participant from Mama Lucy highlights the issue of patient representation at the quality meetings.

"...because at times we might be mistreated by the staff but keep quiet because we don't have representatives. So, we need a representative of the patients who will speak on our behalf." Source: Mama Lucy Kibaki Hospital FGD participant

Influence of Quality Improvement on Prevention of Mother To Child Transmission

The KII respondents cited quality improvement as an essential aspect of PMTCT service delivery, mainly because it helps in improving service delivery. This is because the HCWs can use it to identify gaps in service delivery and address them continuously while monitoring the progress made. Quality improvement enhances safety, effectiveness, and efficiency in service delivery. Through quality improvement, the facility teams have had some benefits, including reduction in MTCT rates, reduced stigma among clients, increased retention rates, and patient outcomes due to improved services, as well as increased vigilance among HCWs. The facilities were implementing QI projects, which ranged from projects tracking viral load uptake and suppression (Mama Lucy), increasing uptake of partner and family testing (Westlands), and increasing IPT uptake (Kangemi). All these projects had a duration of six months, with the WITs tracking the indicators during data review and WIT meetings.

The review of secondary data from facility registers and DHIS reports showed that the first ANC attendance trends dropped across all the facilities between the two periods, July 2014 to June 2015 and July 2018 to June 2019. All the facilities, except Kangemi HC, where there was an increase in the absolute numbers 
of patients tested for HIV, had a noticeable decrease in the number of tests done at ANC between the two periods. The HIV positivity (yield) among the mothers attending ANC dropped across all the facilities with $95 \%$ ART initiation rates across the board.

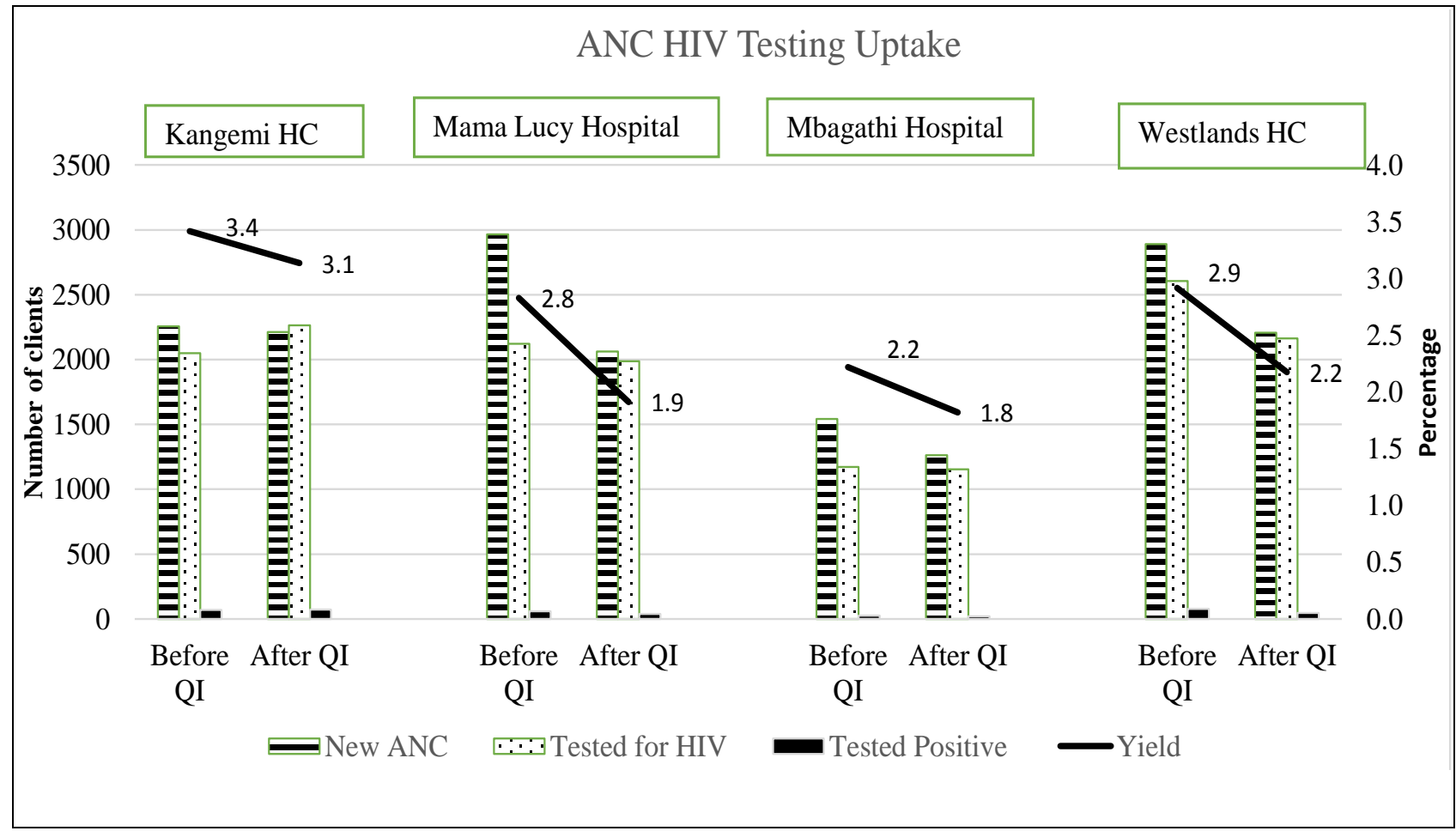

Figure 3: The HIV Services Uptake at the Antenatal Clinic; a comparison of before and after the Quality Improvement interventions

Number of PCR tests done: Except for Westlands HC, where the number of PCR tests dropped, all the other sites had an increase in the number of PCR between the two periods. However, the transmission rates increased in all the facilities.

\section{Early Infant Diagnosis (PCR) within 2 months}

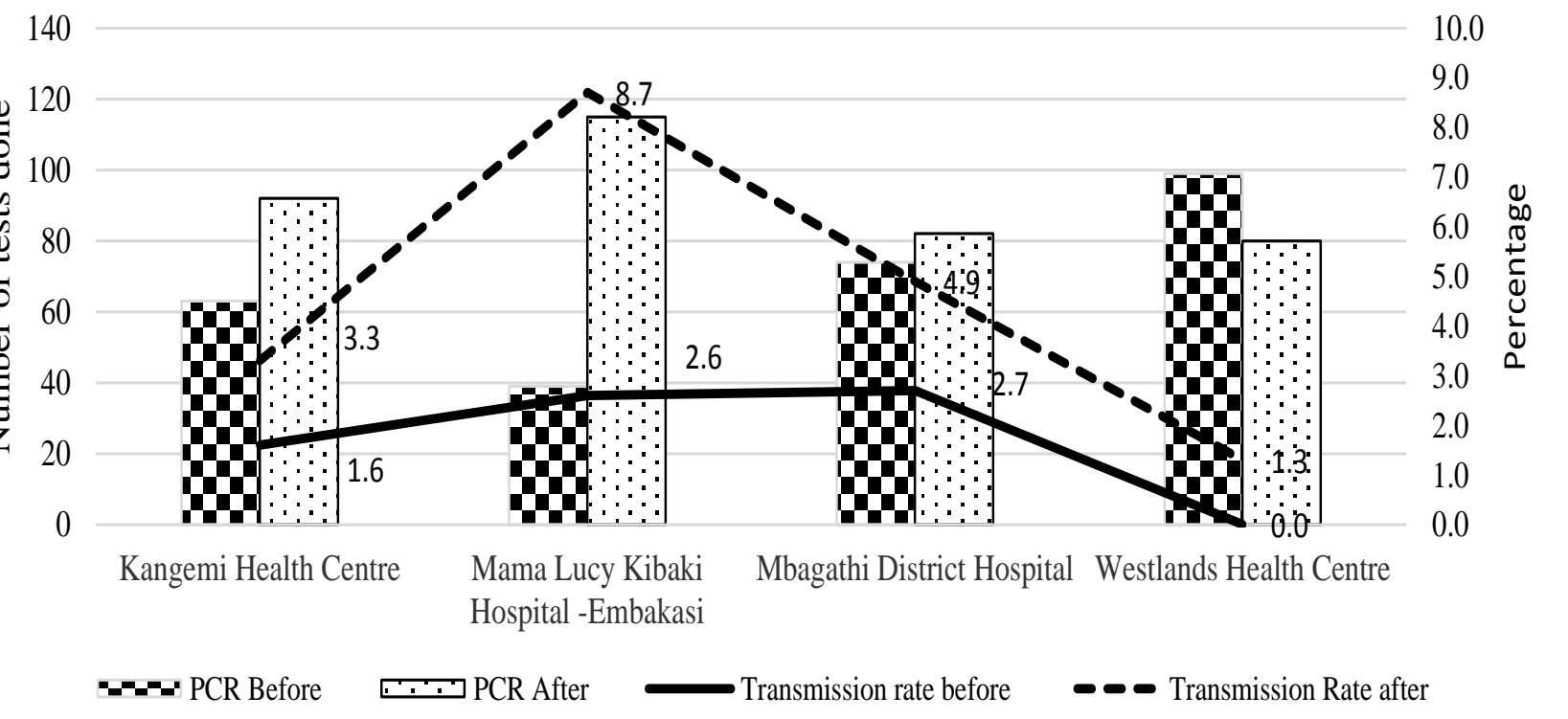

Figure 4: The Early Infant Diagnosis (PCR) uptake within 2 months: a comparison of before and after Quality Improvement Interventions 


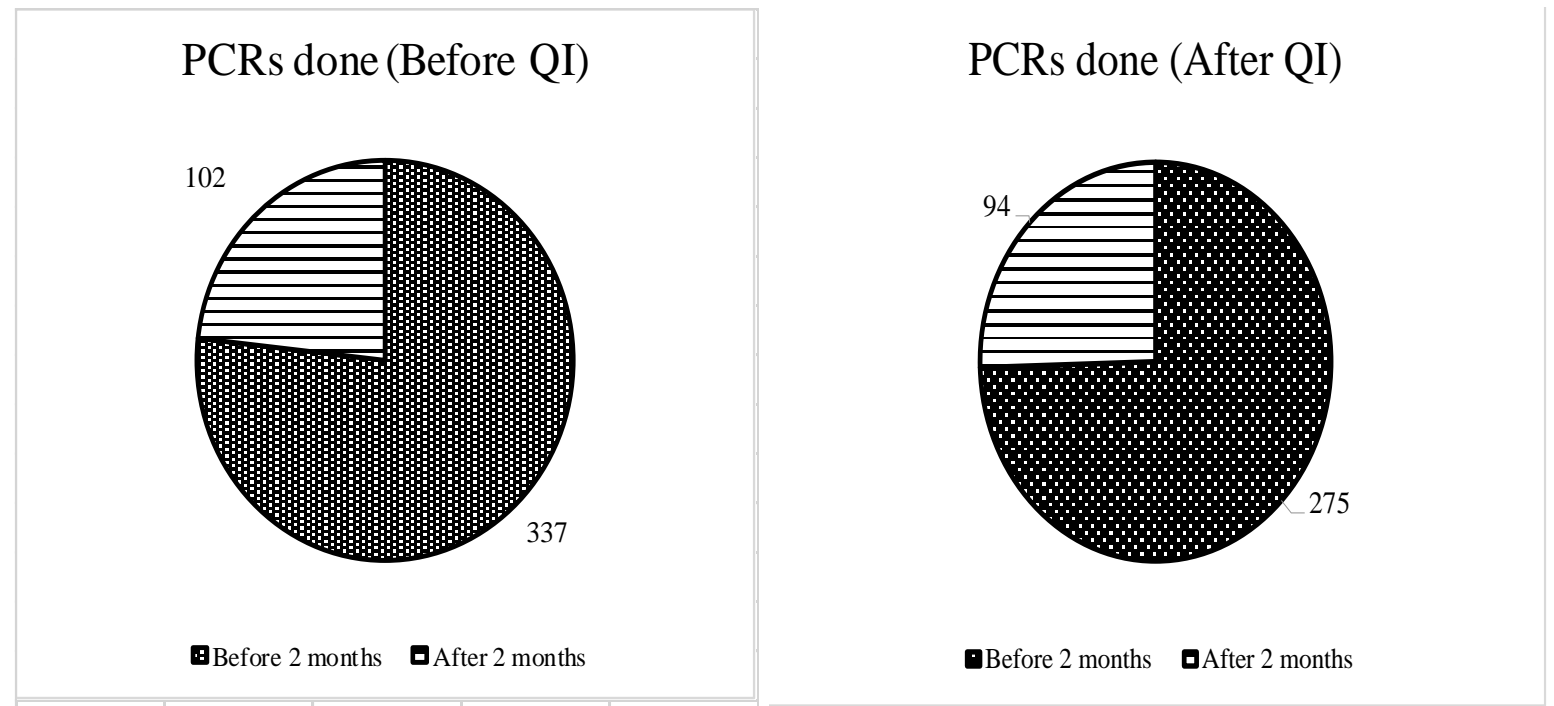

Figure 5: Overall PCR uptake: a comparison of before and after Quality Improvement Interventions

The percentage of HEIs receiving PCR for early infant diagnosis did not vary between the two periods i.e., 77\% (337 PCR samples out of 439) done within 2 months in the period before QI implementation against 75\% (275 PCR samples out of 369) done in the period after QI implementation.

\section{Association between variables}

A cross-tabulation to check the overall uptake of HIV testing services before and after QI implementation showed significant difference with a chi-square test of 1050.126 with a $\mathrm{p}$ value of 0.00001 at 0.05 level of significance. A cross-tabulation to check early infant diagnosis within two months of birth for the period before and after QI implementation showed that there was no significant difference with a chi-square statistic of 0.8122 with a pvalue of 0.367482 at 0.05 level of significance.

\section{Influence of Quality Improvement on Retention to Care}

The secondary data review showed unavailability of data on 24month retention (PMTCT completion) in the period before 2016. However, the introduction of the maternal cohort analysis provided an insight into the retention rates for PMTCT mothers from 2016, and it is in use in the facilities to track retention. The graph below represents the retention trends for mothers enrolled in PMTCT in 2016 to 2017 and completing the PMTCT program in 2018 to 2019. The results show an increase in the absolute numbers of PMTCT clients enrolled and retained up to the completion of the PMTCT program.

\section{Retention in PMTCT}

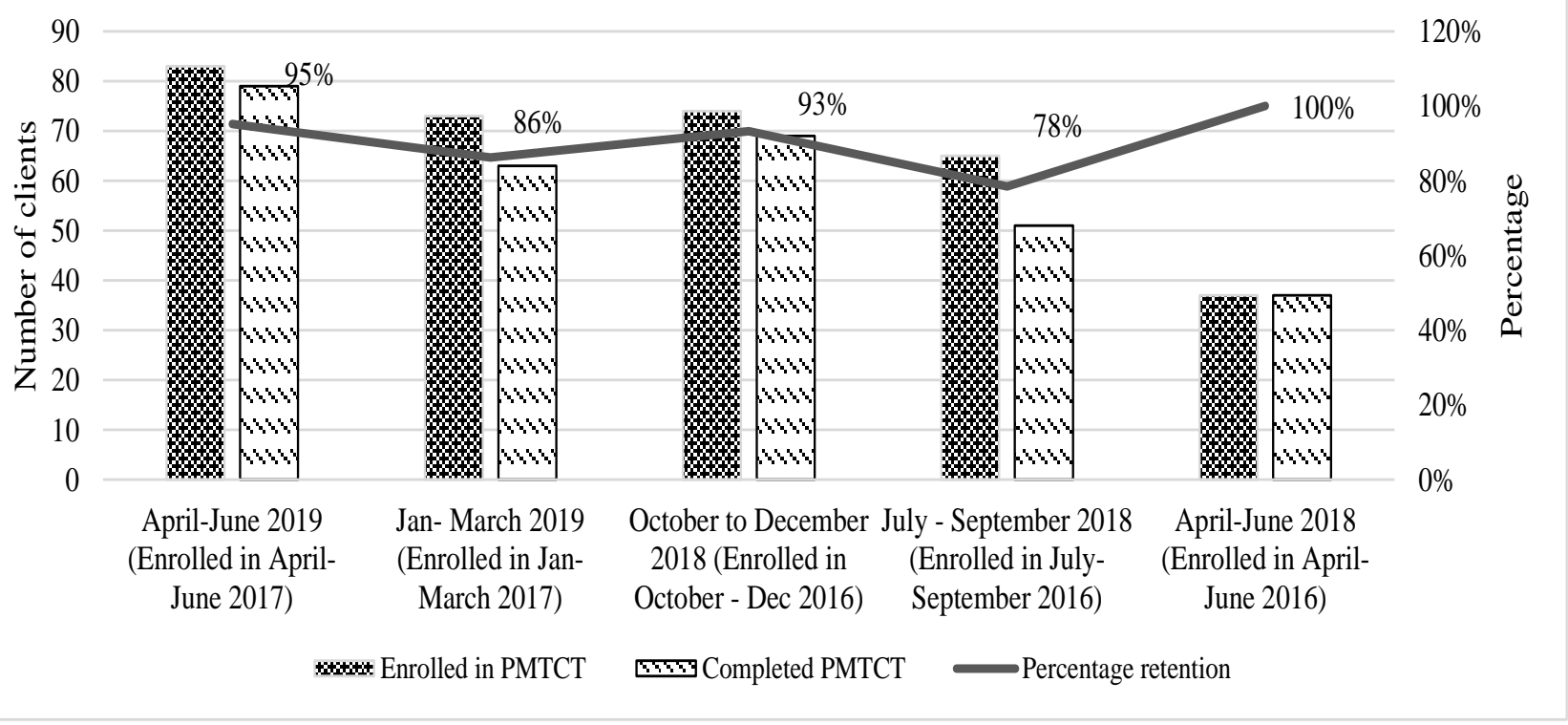

Figure 6: Retention of the mother-baby pair in PMTCT: a comparison between the numbers enrolled and those retained up to the end of 24 months

The focus group discussions brought forth key insights into the clients, perception and experience as they access PMTCT services in the different facilities. The services offered to pregnant and breastfeeding women fall under the following six categories: triaging, counselling and testing services for adults and children, support groups, drug administration, family planning services for mothers, and lessons on nutrition. 
When analysing why the mothers decided to access the services at the facilities, several cross-cutting themes emerge. First, many patients came to the facility because they were pregnant. Once at the clinic, they were tested and diagnosed to be positive, after which they received PMTCT services available at the facilities. Various reasons made them stick with the said facilities, including proximity of the health facility to their places of living, guaranteed health care from qualified staff, availability of drugs, and caring staff at the facilities. A few mothers received referrals to the facilities by their spouses and relatives.

On the question of how accessing the services in the given facility been helpful to them, reduction of stigma was a common theme for most of the respondents. Through the counselling services and psychosocial support offered at the facilities, many respondents said they learned to accept themselves and their HIV status, as well as how to protect their babies from contracting HIV. The drugs offered made the mothers [and fathers] to live positively while ensuring that their children are born and stay negative of the HIV virus. Beyond the PMTCT services, the clients also accessed additional services such as family planning, routine immunization and weight monitoring for the babies. Most FGD participants could point out the relationship between PMTCT and the prevention of passing the HIV virus to unborn babies.

Regarding what they liked about the services, the respondents spoke about the facilities and the staff members at the facilities. Concerning the facilities, most participants liked that the facilities were clean, drugs are available, and that patients' records were up to date. Most participants also appreciated the fact that they received free quality services. The staff at all the facilities received a commendation for their professional approach, friendly and understanding demeanour, and the fact that most treated the patients equally and helped in stigma mitigation. This is as highlighted by the following quote from a Kangemi FGD participant:

"If you are afraid to talk to your husband about your status, they help you talk to him..." Source: Kangemi Health Centre FGD participant

Regarding whether they would recommend the facility to their family or friends, the participants were very positive. The respondents cited good quality services, friendly and welcoming staff, fair treatment, and cleanliness of the facilities as reasons for the positive recommendations. On the other hand, delayed service and undue favouritism were the reasons for the negative recommendations. At Mama Lucy, respondents decried the issue of privacy for PMTCT mothers, as seen below:

"I also feel that for us who are positive we should have our specific place..."

"Yes, because sometimes there are some people who come here, and we go to the same church and we feel like they might know our condition." Source: Mama Lucy Kibaki Hospital FGD participants

\section{Discussion}

Factors Influencing Implementation of Quality Improvement Approaches

The study established that all the sites under study had several factors influencing the implementation of quality improvement approaches, which involve both facility and client factors. Some of these factors are the identified gaps in service delivery, Staff shortage and heavy workload limiting the ability to conduct meetings, lack of co-operation between the teams (staff and clients) affecting teamwork as well as client involvement in QI. In addition, the presence of support from facility management and availability of data to identify performance gaps as well as monitor performance against targets influenced QI. This is consistent with other studies done in Kenya showing challenges in QI implementation that involved the use of both primary and secondary sources of data to make conclusions and targeted health care providers ${ }^{[17]}$. The success of QI implementation is dependent on the identification of strategies to mitigate the gaps identified during implementation.

At the facility level, participation by the management on QI is a vital factor because they are responsible for providing oversight and leadership on quality improvement and bringing the whole facility teams on board with QI. The management is key in providing support supervision, ensuring adequate supplies and commodities for service provision, ensuring that staff are capacity built and receive regular updates in their service areas, providing good governance as well as continuous monitoring and reviewing QI projects. The level of management involvement in QI may be influenced by the position held, as those in senior positions may have more responsibilities within the facility, thereby task shifting the QI process to other levels of staff. This, however, does not negate the importance of management involvement in QI. For any QI initiative to be successful, management support is crucial. This is consistent with other studies where the involvement of management was vital in the success of Quality Improvement ${ }^{[32]}$.

The level of teamwork among the members of the work improvement team (WIT) also affects the implementation of QI. The WIT is normally comprised of different cadres of staff whose input is crucial for quality improvement. The teams have to be committed and have to meet regularly to be able to identify and address gaps in service delivery as part of the CQI process. This team should also include clients whose perspective is key and can provide valuable insights in improving the quality of services that they receive. With this feedback, the facility can implement services that cater to the needs of the client, while ensuring adherence to the minimum standards of patient care. It is noteworthy that other studies demonstrated the importance of teamwork and commitment to the QI process. Continued capacity building, regular meetings, and engagement served contributed to the successful implementation of QI in these study areas ${ }^{[1,3,18]}$.

The study also found out that the availability of accurate data to monitor the performance of the indicators under review affects quality improvement. It is not possible to monitor improvement without data, which should undergo periodic review to identify and address gaps. This forms part of the process in CQI. This finding concurs with studies done showing consistent data use in QI implementation in other countries. Quality Improvement was used by the teams to improve the completeness and accuracy of data that is used to monitor the PMTCT program ${ }^{[5,15]}$. The study facilities use standard tools to monitor the QI process which include HIV-exposed infant cards, viral load tracking register, data abstraction tools, and minutes taken during the work improvement meetings. In addition, the facilities use run charts, PDSA graphs, client exit, forms and data review minutes to monitor the QI process. This is in line with the recommendations made in KHQIF [14].

Influence of Quality Improvement on Prevention of Mother To Child Transmission Outcomes

The study established that the implementation of quality improvement approaches has improved PMTCT service delivery, 
as shown from the trends in the performance indicators and client feedback. The HCWs are committed to ensuring that they identify and address gaps in service delivery through CQI, all to ensure that they are taking care of their clients' satisfaction. The study found out that all four facilities offer a standard package of care consistent across the board, which is in line with the recommendations in the PMTCT guidelines ${ }^{[29]}$.

The decline in numbers of women getting pregnant contributed to the reduction in trends in the first ANC visits. However, the uptake of HIV testing among the clients in the first ANC visit has improved with reduced missed opportunities signifying an improvement in the package of services offered. This is consistent with national and county trends ${ }^{[22]}$. The yield in HIV positivity also reduced, with all newly diagnosed clients started on ART in line with the guidelines. This signifies that the facilities under study offer services that are in line with the guidelines and strive to ensure its application when offering PMTCT services. The trends in mother to child transmission did increase over the period, which might be due to clients accessing care later in their pregnancy. Late access to care delays diagnosis and subsequent treatment prophylaxis for both mother and child. These findings were consistent with the national figures ${ }^{[22]}$. Additionally, QI implementation has led to the consistent use of data to track the performance of PMTCT indicators. The availability of quality data has ensured that the facilities have an opportunity to implement data-informed interventions. In this way, they can cater to the needs of their clients.

\section{Influence of Quality Improvement on Retention to Care}

Customer satisfaction is the most important aspect of quality health care ${ }^{[7]}$. It is key determinant of whether a client will return to a facility for service delivery. Having integrated services for the mother-baby pairs in which they receive services as a one-stopshop fosters retention. Other studies have shown the same findings on the integration of services and retention where the mother-baby pairs were more likely to be retained where they receive PMTCT interventions with integrated services ${ }^{[26]}$. The study established that the quality of PMTCT services offered across the four facilities under study generally satisfy the clients needs. These clients would gladly recommend the services to their family and friends. Services across the four study facilities are integrated and the mother can get all the required services at a go, which improves efficiency and retention. Having a one stop shop for the clients is key in ensuring that services are provided in a way that meets their needs and ensures customer satisfaction. Data on retention seems to corroborate this as the facilities have good retention rates across the board. The presence of quality services, and customer satisfaction is what was able to motivate the clients to keep attending the clinic, hence the good retention rates. The results also show that healthcare providers' attitude as they offer services to clients affects retention. These results are consistent with those found in other studies where the healthcare workers who took part in quality improvement consistently strove to better themselves while providing services through changing their attitude ${ }^{[3]}$. The staff at all the study facilities received a commendation for their professional approach, friendly and understanding demeanour, and the fact that most treated the patients equally and helped in stigma mitigation. The facility environment affects the clients' decision to access care at a particular facility, with clean and well-organized facilities being favoured over the less clean ones. The reduction in stigma through the counselling services and psychosocial support offered at the facilities was also key in fostering retention.

\section{Study Limitations}

The researcher chose the facilities purposefully based on an inclusion and exclusion criteria that is restrictive in nature. Due to this, the study findings may not be extrapolated or generalized to other health care facilities in Nairobi County. The researcher based the study on a review of reported data, which may be subject to errors based on the process of data capture in the original documents. Also, some records may have been misplaced. In this case, the researcher relied on information reported in the DHIS. The PMTCT indicators in the DHIS underwent revision in 2016 to include some missing indicators in the earlier versions. All the four facilities had consistently been reporting in the DHIS during the period in question, with reporting improving after Quality Improvement implementation. There may, however, been some information bias based on data entry errors. The study relied on the participants' opinions discussed in a group setting. This made it susceptible to having the views of some participants dominating the conversation and limiting the interaction with the quiet ones. However, the study reduced this by ensuring that the research assistants had the required expertise in moderating focus group discussions.

\section{Conclusions}

Implementing QI in PMTCT in the study facilities has led to efficient services, customer satisfaction, and improved outcomes. Through QI, the HCWs strive to offer a comprehensive package of services that cater to the clients' needs while ensuring good patient outcomes for both the mother and baby. From the feedback received from the clients, the attitude of the health care workers towards them influences whether they would access services in a particular facility. Additionally, the situation of the environment also determines accessibility and retention. The clients showed satisfaction with services offered to them, which means that QI implementation has positively affected service delivery. To enhance the work of the QI team, it is essential to involve consumers in the process of QI implementation. This is because the consumers provide valuable feedback on the healthcare delivery experience, revealing the areas that would benefit from improvement. Their ideas on improvement are very different from the ones presented by the health workers. Client involvement is key to ensuring customer satisfaction. The success of QI implementation relies on having support and participation from the facility management and teams who are committed to the process. Additionally, completeness and accuracy of data allows for performance monitoring and is crucial in demonstrating the improvement of the services offered.

\section{Ethics approval and consent to participate}

The Kenyatta National Hospital- University of Nairobi (KNHUoN) Ethics Committee granted the authority to conduct the study. Approval was also sought from Nairobi County research board and National Commission for Science Technology and Innovation (NACOSTI). The local Sub County Medical Officer of Health (SCMOH), and the facility in-charges, were also informed and their consent sought. Informed consent was sought from the research participants before commencing the data collection. The researcher sought informed consent from the respondents after explaining the purpose and importance of the study. The researcher briefed the participants in the FGDs on the intention to use a voice recorder during the discussions, allowing them to choose participation. For the maintenance of confidentiality, the study did not record any participants' names or identification features. 


\section{List of abbreviations}

$\begin{array}{ll}\text { AIDS } & \text { Acquired Immunodeficiency Syndrome } \\ \text { ANC } & \text { Ante Natal Care } \\ \text { ARV } & \text { Antiretroviral } \\ \text { ASSIST } & \text { Applying Science to Strengthen and Improve } \\ & \text { Systems } \\ \text { AZT } & \text { Zidovudine } \\ \text { CD4 } & \text { Cluster of Differentiation 4 } \\ \text { DHIS } & \text { District Health Information System } \\ \text { EID } & \text { Early Infant Diagnosis } \\ \text { EMTCT } & \text { Elimination of Mother to Child Transmission } \\ \text { FGD } & \text { Focus Group Discussion } \\ \text { FP } & \text { Family Planning } \\ \text { HAART } & \text { Highly Active Anti -Retroviral Therapy } \\ \text { HEI } & \text { HIV Exposed Infant } \\ \text { HIV } & \text { Human Immunodeficiency Virus } \\ \text { IHI } & \text { Institute for Healthcare Improvement } \\ \text { KDHS } & \text { Kenya Demographic Health Survey } \\ \text { KEPH } & \text { Kenya Essential Package of Health } \\ \text { KII } & \text { Key Informant Interview } \\ \text { KQM } & \text { Kenya Quality Model } \\ \text { KQMH } & \text { Kenya Quality Model for Health } \\ \text { KHQIF } & \text { Kenya HIV Quality Improvement Framework } \\ \text { MOH } & \text { Ministry of Health } \\ \text { MTCT } & \text { Mother to Child Transmission } \\ \text { NASCOP } & \text { National AIDS and STI Control Program } \\ \text { NVP } & \text { Nevirapine } \\ \text { PDSA } & \text { Plan Do Study Act } \\ \text { PEPFAR } & \text { U.S President's Emergency Plan for AIDS Relief } \\ \text { PHFS } & \text { Partnership for HIV Free Survival } \\ \text { PMTCT } & \text { Prevention of Mother to Child Transmission } \\ \text { QI } & \text { Quality Improvement } \\ \text { QIT } & \text { Quality Improvement Team } \\ \text { TB } & \text { Tuberculosis } \\ \text { TQM } & \text { Total Quality Management } \\ \text { UNAIDS } & \text { United Nations Program on HIV/AIDS } \\ \text { UNGASS } & \text { United Nation General Assembly Special Session } \\ \text { UNICEF } & \text { United Nations Children's Fund } \\ \text { USAID } & \text { United States Agency for International } \\ \text { WHO } & \text { Development } \\ \text { WIT } & \text { World Health Organization } \\ \text { WTC } & \text { Lamivudine } \\ & \end{array}$

\section{Data Availability}

Data will be made available upon request and after publication

\section{Conflicts of Interest}

The authors declare that there is no conflict of interest regarding the publication of this paper.

\section{Funding Statement}

The authors did not receive any funding for this work

\section{Authors' contributions}

DNM came up with the concept. CB analysed and interpreted the result. PM provided overall guidance and review. All were major contributors in writing the manuscript. All authors read and approved the final manuscript

\section{References}

[1] Barker, P., Barron, P., Bhardwaj, S., Pillay, Y., 2015. The role of quality improvement in achieving effective large-scale prevention of mother-to-child transmission of HIV in South Africa. AIDS Lond. Engl. 29 Suppl 2, S137-143. https://doi.org/10.1097/QAD.0000000000000718

[2] Batalden, P.B., Davidoff, F., 2007. What is "quality improvement" and how can it transform healthcare? Qual. Saf. Health Care 16, 2-3. https://doi.org/10.1136/qshc.2006.022046

[3] Bradley, J., Igras, S., 2005. Igras S: Improving the quality of child health services: participatory action by providers, in: International Journal for Quality in Health Care 2005. pp. 17-391.

[4] Cock, K.M.D., Fowler, M.G., Mercier, E., Vincenzi, I. de, Saba, J., Hoff, E., Alnwick, D.J., Rogers, M., Shaffer, N., 2000. Prevention of Mother-to-Child HIV Transmission in Resource-Poor Countries: Translating Research into Policy and Practice. JAMA 283, 11751182. https://doi.org/10.1001/jama.283.9.1175

[5] Doherty, T., Chopra, M., Nsibande, D., Mngoma, D., 2009. Improving the coverage of the PMTCT programme through a participatory quality improvement intervention in South Africa. BMC Public Health 9, 406. https://doi.org/10.1186/1471-2458-9-406

[6] Donabedian, A., 2002. An Introduction to Quality Assurance in Health Care. Oxford University Press.

[7] Gupta, K.S., Rokade, V., 2016. Importance of Quality in Health Care Sector: A Review. J. Health Manag. 18, 8494. https://doi.org/10.1177/0972063415625527

[8] Hashmi, K., 2013. Introduction and Implementation of Total Quality Management (TQM). URL https://www.isixsigma.com/methodology/total-qualitymanagement-tqm/introduction-and-implementation-totalquality-management-tqm/ (accessed 3.22.18).

[9] Health Resources and Services Administration, 2011.

[10] IOM, 2018. Crossing the Quality Chasm: The IOM Health Care Quality Initiative: Health and Medicine Division [WWW Document]. URL http://www.nationalacademies.org/hmd/Global/News\%2 0Announcements/Crossing-the-Quality-Chasm-TheIOM-Health-Care-Quality-Initiative.aspx （accessed 3.20.18).

[11] Kayongo, M., Butera, J., Mboninyibuka, D., Nyiransabimana, B., Ntezimana, A., Mukangamuje, V., 2006. Improving availability of EmOC services in Rwanda - CARE's experiences and lessons learned at Kabgayi Referral Hospital. Int. J. Gynecol. Obstet. 92, 291-298. https://doi.org/10.1016/j.ijgo.2005.10.030

[12] KNBS, 2019. 2019 Kenya Population and Housing Census.

[13] MOH, 2019. Kenya Master Health Facility List. URL http://kmhfl.health.go.ke/\#/home (accessed 11.19.19).

[14] MOH, 2014. Kenya HIV Quality Improvement Framework (KHQIF).

[15] Mphatswe, W., S Mate, K., Bennett, B., Ngidi, H., Reddy, J., M Barker, P., Rollins, N., 2012. Improving public health information: a data quality intervention in 
KwaZulu-Natal, South Africa. Bull. World Health Organ. 90, 176-82. https://doi.org/10.2471/BLT.11.092759

[16] Mugenda, O., Mugenda, A., 2008. Research Methods: Qualitative and Quantitative Approaches.

[17] Murungami, R.W., 2014. Challenges Of Implementing Kenya Quality Model For Health Strategy In Kiambu County, Kenya 53.

[18] Mutanda, P., Muange, P., Lutta, M., Kinyua, K., Chebet, L., Okaka, B., Mwamzandi, Y., Mwavita, J., 2017. Improving prevention of mother to child transmission of HIV care: Experiences from implementing quality improvement in Kenya 45.

[19] Mwaniki, M.K., Vaid, S., Chome, I.M., Amolo, D., Tawfik, Y., Kwale improvement coaches, 2014. Improving service uptake and quality of care of integrated maternal health services: the Kenya kwale district improvement collaborative. BMC Health Serv. Res. 14. https://doi.org/10.1186/1472-6963-14-416

[20] NASCOP, 2012. Guidelines for Prevention of Mother to Child transmission (PMTCT) of HIV in Kenya, 4th edition.

[21] NASCOP HIV QI, 2018. . NASCOP. URL http://www.nascop.or.ke/?page_id=2324 (accessed 3.21.18).

[22] NASCOP, NACC, 2018. Kenya HIV Estimates.

[23] NASCOP, NACC, 2016. Kenya HIV County Profiles.

[24] Padian, N., McCoy, S., Karim, A., Hansen, N., Kim, J., Bartos, M., Katabira, E., Bertozzi, S., Shwartlander, B., Cohen, M., 2011. HIV prevention transformed: the new prevention research agenda - The Lancet [WWW Document].

URL http://www.thelancet.com/pdfs/journals/lancet/PIIS01406736(11)60877-5.pdf (accessed 3.21.18).

[25] Schroeder, R.G., Linderman, K., Zhang, D., 2009. Evolution of Quality: First Fifty Issues of Production and
Operations Management. Prod. Oper. Manag. 14, 468481. https://doi.org/10.1111/j.1937-5956.2005.tb00234.x

[26] Tudor Car, L., van-Velthoven, M.H., Brusamento, S., Elmoniry, H., Car, J., Majeed, A., Atun, R., 2011. Integrating prevention of mother-to-child HIV transmission (PMTCT) programmes with other health services for preventing HIV infection and improving HIV outcomes in developing countries, in: The Cochrane Library. John Wiley \& Sons, Ltd. https://doi.org/10.1002/14651858.CD008741.pub2

[27] USAID ASSIST, 2015. Kenya Quality Model for Health: A Training Course for the Health Sector | USAID ASSIST Project [WWW Document]. URL https://www.usaidassist.org/resources/kenya-qualitymodel-health-training-course-health-sector (accessed 3.21.18).

[28] Wamuyu, G.M., 2015. Total Quality Management in Kenya's Healthcare Industry 65.

[29] WHO (Geneva), 2010. PMTCT strategic vision 20102015 preventing mother-to-child transmission of HIV to reach the UNGASS and Millennium development goals: moving towards the elimination of paediatric HIV. World health organization (WHO), Geneva.

[30] World Health Organization, 2007. Everybody's business: strengthening health systems to improve health outcomes : WHO's frmaework for action. World Health Organization, Geneva.

[31] Wouters, A., 1995. Improving quality through cost recovery in Niger. Health Policy Plan. 10, 257-270. https://doi.org/10.1093/heapol/10.3.257

[32] Youngleson, M.S., Nkurunziza, P., Jennings, K., Arendse, J., Mate, K.S., Barker, P., 2010. Improving a Mother to Child HIV Transmission Programme through Health System Redesign: Quality Improvement, Protocol Adjustment and Resource Addition. PLOS ONE 5, e13891. https://doi.org/10.1371/journal.pone.0013891 